\title{
面向实物交互的空间增强现实数字孪生法配准技术
}

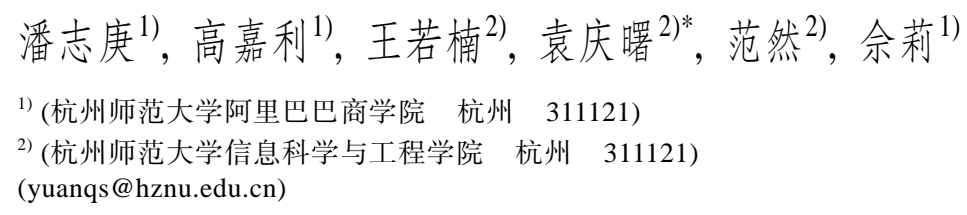

摘 要: 在支持实物交互的空间增强现实系统中, 交互会引起实物对象方向、位置的快速改变. 为了确保实物移动过 程中的虚实配准实时性和精确性, 提出一种面向实物交互的空间增强现实数字孪生法配准技术. 通过在数字空间建 立与物理空间一致的对象数字模型，实时捕获物理空间中对象的方向、位置信息，利用该信息实时调整数字空间中的 虚拟对象方向、位置参数; 离线标定投影机的内外部参数，同时在数字空间中建立与物理空间中投影机内外部参数一 致的虚拟相机, 将实时渲染生成的数字图像输出至投影画面, 从而实现虚实图像的精确配准. 使用搭建的投影式交 互桌面开展中学物理认识磁感线实验, 交互过程中磁铁、指南针的检测与配准结果表明, 该技术具有很好的配准实时 性和精度, 能够满足空间增强现实系统中的实物交互需求.

关键词：空间增强现实；投影着色；虚实配准；实物交互

中图法分类号: TP391.41 DOI: 10.3724/SP.J.1089.2021.18556

\section{Digital Twin Registration Technique of Spatial Augmented Reality for Tangible Interaction}

\author{
Pan Zhigeng ${ }^{1)}$, Gao Jiali ${ }^{1)}$, Wang Ruonan ${ }^{2)}$, Yuan Qingshu ${ }^{2 *}$, Fan $\operatorname{Ran}^{2)}$, and She $\mathrm{Li}^{1)}$ \\ ${ }^{1)}$ (Alibaba Business School, Hangzhou Normal University, Hangzhou 311121) \\ ${ }^{2)}$ (School of Information Science and Engineering, Hangzhou Normal University, Hangzhou 311121)
}

\begin{abstract}
In spatial augmented reality (SAR) systems supporting tangible interaction, user interactions can cause rapid changes in orientation and position of interaction objects. To ensure the efficiency and accuracy of registration during objects movements, a digital twin registration technique for SAR is proposed. Models are made in the digital space and their geometric parameters are entirely consistent with the objects in the physical space. The orientation and position parameters of the physical objects are tracked in real-time during user interaction. Then the digital objects are adjusted according the parameters. Moreover, intrinsic and extrinsic parameters of the projector, which are calibrated in advance, are used to set the virtual camera in the digital space. The projection patterns are rendered in that virtual camera and then projected onto the interaction objects. The efficiency and accuracy are evaluated in the middle school physical experiment learning magnetic induction line based on the projection interaction tabletop, which meet the requirements of tangible interaction in SAR systems.
\end{abstract}

Key words: spatial augmented reality; projection mapping; virtual-real registration; tangible interaction

收稿日期：2020-08-06; 修回日期：2020-09-30. 基金项目：国家重点研发计划(2018YFB1004901); 浙江省自然科学基金 (LY19F020020). 潘志庚(1965一), 男, 博士, 教授, 博士生导师, CCF 会员, 主要研究方向为增强现实、人机交互; 高嘉利(1996一), 女, 硕士研究生, 主要研究方向为增强现实、人机交互; 王若楠(1996一), 女, 硕士研究生, 主要研究方向为增强现实、人机交互; 袁庆曙(1979-), 男, 博士, 讲师, CCF 会员, 论文通讯作者, 主要研究方向为增强现实、人机交互; 范然(1984一), 男, 博士, 讲师, 主要研究方向为计算机图形图像、三维几何测量; 余莉(1978一), 女, 博士, 讲师, 主要研究方向为隐私保护、数据挖掘. 
增强现实根据显示装置位置不同，可以分为 头戴式增强现实、手持式增强现实和空间增强现实 三大形式 ${ }^{[1]}$. 空间增强现实作为增强现实的 3 种重 要形式之一, 利用投影机等装置将虚拟内容呈现 于物理空间 ${ }^{[2]}$, 达到虚实融合的效果. 与另外 2 种 形式的增强现实系统相比，空间增强现实具有独 特的优势. 首先, 用户可以专注于物理空间中的任 务, 系统使用时的认知负荷较低; 其次, 用户不需 要佩戴或携带增强现实显示设备, 没有交互负载; 再次, 由于在物理空间中直接呈现, 多个用户可以 同时从不同方位观察到虚实融合的内容, 支持群 体式的增强现实体验 ${ }^{[3]}$.

正是由于上述优势，空间增强现实技术近年 来逐渐被广泛地应用于多个领域. 在空间增强现 实系统中, 用户可以在物理空间中与实物进行自 然交互，交互对象可作为输人和输出信息的双载 体, 能实现交互输人与视觉呈现的完全一致化, 给 用户带来了良好的交互体验. 然而, 实物交互 ${ }^{[4]}$ 的 引人，对空间增强现实的虚实配准提出了新的挑 战. (1) 实时性. 由于交互对象在快速移动, 因此 虚拟内容需要根据交互信息做实时改变与调整. (2) 配准精度. 由于有实物对象参与交互, 必须有 较高的配准精度, 才能实现实物对象、物理场景、 虚拟内容的精确吻合.

目前，空间增强现实的虚实配准主要有以下 方法.

（1）基于硬件跟踪设备的配准. 其原理主要是 通过电磁、光学等跟踪器硬件直接获取相机与实物 对象的位置与方向(位姿或姿态)数据, 从而实现增 强的数字信息实时注册. Bandyopadhyay 等 ${ }^{[5]}$ 利用 电磁跟踪器跟踪实物对象及画刷, 用户可以用画 刷在实物对象上绘画，绘画内容通过多台投影机 实时投影画面. 他们同时还使用了光学跟踪器进 行对比, 发现使用电磁跟踪器时延迟小, 可全向跟 踪. Marner 等 ${ }^{[6]}$ 使用光学跟踪器跟踪画笔及实物对 象. 在实物对象上实时绘制出设计图案. 由于基于 硬件跟踪器的配准需要在交互时绑定一定的硬件 装置, 在开展虚拟实验时, 会给用户带来一定的交 互负载.

（2）基于视觉的配准. 通常是利用计算机视觉 跟踪对象, 并以此恢复场景或对象的位姿, 最终计 算配准参数进行虚实配准, 它是近年来常用的空 间增强现实配准手段. 根据技术手段的不同，又可 以分为基于标签的配准、基于自然特征的配准以及 基于深度相机的配准. 基于标签的跟踪识别相对
有比较好的稳定性, 如 Khan 等 ${ }^{[7]}$ 设计了高质量 ARToolkit 标签的生成方法; Fiala ${ }^{[8]}$ 开发了 ARTag 标签. Attard 等 ${ }^{[9]}$ 设计了 TangiBoard 系统, 该系统 使用相机检测白板上实物对象的黑白标签, 根据 标签使用的语义, 交互投影数字内容. Kern 等 ${ }^{[10]}$ 利 用相机识别对象周围的黑白标签, 间接地得到与 相机捆绑的投影机的位姿，将带有纹理信息的内 容投影到实物对象上, 用于辅助三维扫描数据的 质量检查. Narita 等 ${ }^{[11]}$ 提出了一种可用于形变表面 的点簇标签, 并应用于纸张、布料等的空间增强显 示. Kagami 等 ${ }^{[12]}$ 将标签嵌人投影画面中, 利用同 步的高速相机拍摄进行跟踪配准. 基于自然特征 的配准主要是通过识别对象本身的纹理特征进行 跟踪配准. 由于受投影光照的影响, 在空间增强现 实中并不多见. 基于深度相机的配准方法主要是 基于深度图中的几何结构信息重建对象的位姿, 并进行虚实配准. Kundu 等 ${ }^{[13]}$ 设计的 AR 沙箱根据 沙子表面的线条投影呈现出实际的地形和水流等 信息, 但由于沙箱的几何结构不显著, 其配准精度 需求相对较低. Kurth 等 ${ }^{[14]}$ 提出了一个由目标对 象、多个投影机和基于深度的跟踪系统共同组成的 动态多投影映射系统, 提高特征点较少时的检测 跟踪算法效率, 在标定阶段获得相对准确的投影 机内外参数, 完成在任意曲面上进行虚实配准. 该 方法虽然都提到实物对象可以移动, 移动后会自 动优化配准，但并未提及将对象用于实时交互.

(3) 基于硬件跟踪及视觉的混合配准. 由于单 一来源的跟踪数据可能会有不稳定问题, 也有将 多种跟踪数据混合进行配准的方法. Pratticò 等 ${ }^{[15]}$ 混合三自由度加速度传感器及基于标签的视觉方 法, 定位实物交互对象的位姿, 从而开展空间增强 的互动游戏. Zhou 等 ${ }^{[16]}$ 利用深度相机得到的点云 进行对象的位姿识别与配准, 在配准有延迟或不 稳定时, 使用 AHRS 航姿参考系统进行跟踪, 最终 实现了三维对象的快速投影上色.

\section{1 配准算法}

本文提出了一种面向实物交互的空间增强现 实数字孪生法配准技术, 在数字空间建立与物理 空间一致的对象模型, 并根据物理空间的变化, 实 时调整数字空间的对象参数. 同时，在数字空间建 立与物理空间中的投影机内外部参数一致的虚拟 相机, 进行虚拟内容的渲染生成. 最后, 把投影机 当作一个逆向相机，将实时绘制的图像投影到真 
实场景中，从而实现虚拟内容与真实场景的精确 配准.

上述思想借鉴了数字孪生 ${ }^{[17]}$ 的概念，该概念 由密歇根大学教授 Grieves 首次提出，在工业中是 指借助于物理模型、传感器更新、运行历史等数据 模拟工业流程，通过在虚拟空间中完成所有部件 的映射，刻画相对应的实体装备的全生命周期过 程. 在本文中指通过在数字空间建立与物理空间 一致的模型，同时赋予数字空间中对象与物理空 间对象一致的参数. 因此，本文将其命名为数字孪 生法配准, 配准的实现流程如图 1 所示.

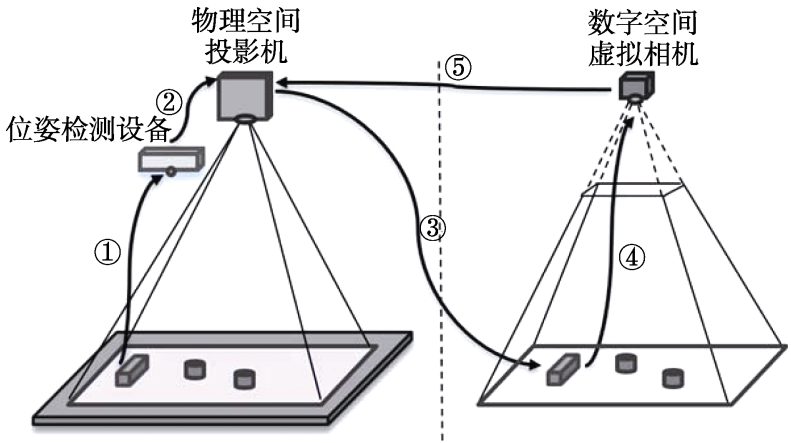

图 1 数字孪生法配准的实现流程

\section{1 检测实物交互对象的位姿}

该技术通过位姿检测设备实时捕获真实场景 中的交互情境, 得到真实物体方向、位置在检测设 备坐标系下的位姿数据. 理论上可以使用各种类 型的跟踪器, 由于空间增强现实中实物交互的需 求，本文采用深度相机实时捕获场景点云，并进行 分析及位姿计算.

\section{2 位姿数据坐标转换}

本文提出的配准技术需将对象在检测设备中 的坐标, 转化至投影机坐标系下的三维坐标. 其中 位姿检测设备下的物体坐标与投影机坐标系下的 坐标转换关系可由一个旋转变换矩阵 $\boldsymbol{R}$ 和一个平 移变换向量 $\boldsymbol{T}$ 表示，假设投影机坐标系下三维点 为 $P_{1}\left(X_{\mathrm{p}}, Y_{\mathrm{p}}, Z_{\mathrm{p}}\right)$, 该点在位姿检测设备坐标系中 对应点为 $P_{2}\left(X_{\mathrm{c}}, Y_{\mathrm{c}}, Z_{\mathrm{c}}\right)$, 则有转换关系

$$
\left[\begin{array}{lll}
X_{\mathrm{p}} & Y_{\mathrm{p}} & Z_{\mathrm{p}}
\end{array}\right]^{\mathrm{T}}=\boldsymbol{R}\left[\begin{array}{lll}
X_{\mathrm{c}} & Y_{\mathrm{c}} & Z_{\mathrm{c}}
\end{array}\right]^{\mathrm{T}}+\boldsymbol{T}
$$

由于本文采用深度相机检测位姿，位姿检测 设备坐标系下的三维坐标 $P_{2}\left(X_{\mathrm{c}}, Y_{\mathrm{c}}, Z_{\mathrm{c}}\right)$ 需要由图 像中的像素点坐标 $P_{i}(u, v)$ 计算得到, 对应关系为

$$
Z_{c}\left[\begin{array}{l}
u \\
v \\
1
\end{array}\right]=\left[\begin{array}{lll}
a_{x} & 0 & u_{0} \\
0 & a_{y} & v_{0} \\
0 & 0 & 1
\end{array}\right]\left[\begin{array}{l}
X_{\mathrm{c}} \\
Y_{\mathrm{c}} \\
Z_{\mathrm{c}}
\end{array}\right]
$$

其中, $a_{x}, a_{y}, u_{0}, v_{0}$ 为深度相机的内部参数. 由式 (1)(2)可知，位姿检测设备所获取图像中像素点坐 标与投影机下三维坐标的矩阵变换关系为

$$
\left[\begin{array}{c}
X_{\mathrm{p}} \\
Y_{\mathrm{p}} \\
Z_{\mathrm{p}}
\end{array}\right]=Z_{\mathrm{c}} \boldsymbol{R}\left[\begin{array}{rrr}
\frac{1}{a_{x}} & 0 & -\frac{u_{0}}{a_{x}} \\
0 & \frac{1}{a_{y}} & -\frac{v_{0}}{a_{y}} \\
0 & 0 & 1
\end{array}\right]\left[\begin{array}{l}
u \\
v \\
1
\end{array}\right]+\boldsymbol{T}
$$

由式(3)便可计算出位姿检测设备所获取图像 中每个像素点在投影机坐标系下的空间位置信息.

\section{3 调整虚拟模型位姿}

将以上步骤检测得到的实物对象的三维位姿 信息转换为投影机坐标系下实物对象的位姿，实 时地调整虚拟场景中虚拟模型的位姿. 当用户直 接操作真实场景中的物体时, 虚拟场景中的物体 也会实时地跟随着变化.

\section{4 虚拟图像渲染生成}

在数字空间中, 根据投影机的内外部参数, 设 置虚拟相机的内外部参数. 控制相机的主要属性 是焦距的大小、镜头位移和传感器的尺寸. 为了保 证虚拟场景中模型通过投影机投影出来的位置和 真实物体位置一致，在虚拟场景中，本文设置虚拟 相机的参数为投影机的参数. 投影机的内参矩阵 可表示为

$$
\boldsymbol{K}=\left[\begin{array}{rrr}
\frac{f}{d_{x}} & 0 & c_{x} \\
0 & \frac{f}{d_{y}} & c_{y} \\
0 & 0 & 1
\end{array}\right] .
$$

其中, $f$ 为投影机的焦距, 单位为 $\mathrm{mm} ; d_{x}$ 和 $d_{y}$ 为 像素方向宽度; $f / d_{x}$ 为使用以像素为单位描述的 $x$ 方向的焦距; $f / d_{y}$ 为使用以像素为单位描述的 $y$ 方向的焦距; $c_{x}$ 和 $c_{y}$ 为投影机光轴在图像坐标 系中的偏移量, 单位为像素.

在物理空间，本文将投影机坐标系作为世界 坐标系, 因此其外部参数可忽略. 同样在数字空间 中，本文也将虚拟相机放置于世界坐标系的原点， 并保持与投影机一致的方向. 利用上述内外部参 数的虚拟相机, 渲染虚拟场景生成投影图像.

\section{5 虚拟图像投影输出}

由于本文根据物理空间中的对象及投影机参 数建立数字空间, 可以将物理空间中的投影机看 做一个逆向的相机，直接投影渲染生成的虚拟图 像, 可以精确配准到物理对象的实体表面. 配准流 程如图 2 所示. 


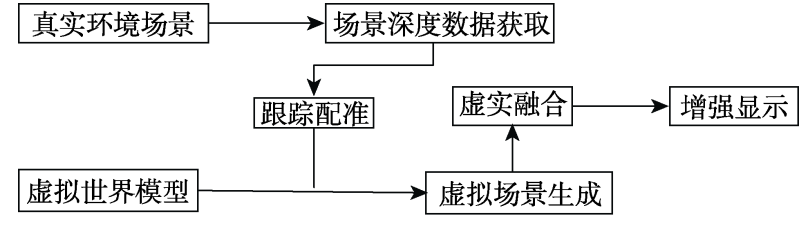

图 2 配准流程

\section{2 实现流程}

本文利用微软 Kinect v2.0 检测实物交互对象 的三维位姿, 基于上述数字孪生法配准技术, 进行 了支持实物交互的空间增强现实系统实现.

\section{1 深度相机坐标系与投影机坐标系的配准}

本文采用 Kinect 传感器中的深度相机对真实 世界中的物体进行检测识别, 将 Kinect RGB 相机 作为系统标定的媒介, 首先利用结构光标定投影 机和 Kinect RGB 相机, 得到其内部参数及外部参 数变换矩阵为

$\left[R_{1} \mid T_{1}\right]=$

$\left[\begin{array}{crrc}-0.9998568 & -0.005632 & -0.053188 & -210.52937 \\ -0.000316 & -0.993800 & 0.111177 & 45.034136 \\ -0.053484 & 0.111034 & 0.992376 & 829.75574\end{array}\right]$.

利用 Matlab 双目相机标定工具箱标定 Kinect RGB 相机和 Kinect 深度相机, 得到其深度相机内部参数 矩阵 $\boldsymbol{K}_{\mathrm{c}}$ 及外部参数变换矩阵 $\left[\boldsymbol{R}_{2} \mid \boldsymbol{T}_{2}\right]$ 分别为

$\left[\boldsymbol{K}_{\mathrm{c}}\right]=\left[\begin{array}{ccc}a_{x} & 0 & u_{0} \\ 0 & a_{y} & v_{0} \\ 0 & 0 & 1\end{array}\right]=\left[\begin{array}{ccc}367.412 & 0 & 254.495 \\ 0 & 367.510 & 205.629 \\ 0 & 0 & 1\end{array}\right]$,

$$
\begin{aligned}
& {\left[\boldsymbol{R}_{2} \mid \boldsymbol{T}_{2}\right]=} \\
& {\left[\begin{array}{lrrr}
0.999923 & -0.012250 & -0.001948 & -51.9256 \\
0.012249 & 0.999925 & -0.000322 & -0.54232 \\
0.001952 & 0.000298 & 0.999998 & -0.28507
\end{array}\right] .}
\end{aligned}
$$

按照以上配准原理中公式推导, 即可得到深 度相机深度图像中像素点 $P_{i}(u, v)$ 到对应投影机坐 标系下三维坐标信息点 $P_{1}\left(X_{\mathrm{p}}, Y_{\mathrm{p}}, Z_{\mathrm{p}}\right)$ 的转换关系

$$
\left[\begin{array}{c}
X_{\mathrm{p}} \\
Y_{\mathrm{p}} \\
Z_{\mathrm{p}}
\end{array}\right]=Z_{\mathrm{c}} \boldsymbol{R}_{1} \boldsymbol{R}_{2}\left[\begin{array}{rrr}
\frac{1}{a_{x}} & 0 & -\frac{u_{0}}{a_{x}} \\
0 & \frac{1}{a_{y}} & -\frac{v_{0}}{a_{y}} \\
0 & 0 & 1
\end{array}\right]\left[\begin{array}{l}
u \\
v \\
1
\end{array}\right]+\boldsymbol{R}_{1} \boldsymbol{T}_{2}+\boldsymbol{T}_{1}(4)
$$

把 Kinect 深度相机获取到的深度数据利用标 定出的转换矩阵经过式(4)进行坐标系的转换, 便 可计算出深度图中每个点在投影机坐标系下的空 间位置信息。

\section{2 虚拟相机参数设置}

本文把投影机视为一个逆向相机, 按照 Unity 相机组件中提供的可以模拟真实相机的设置方式. 投影机的内部参数通过基于编码结构光的标定方 法得到, 标定结果为一个 $3 \times 3$ 的矩阵, 投影机内参 矩阵和具体的设置流程如下.

$$
\boldsymbol{K}=\left[\begin{array}{ccc}
1999.92466 & 0 & 557.841428 \\
0 & 1997.10768 & 25.2043554 \\
0 & 0 & 1
\end{array}\right] .
$$

\subsection{1 焦距的设置}

事实上， $x$ 方向和 $y$ 方向上归一化焦距可以 不同，但在 Unity 中需设置为同一值. 本文设置该 归一化焦距值为 $x$ 和 $y$ 方向的平均值, 即 $\left(f / d_{x}+f / d_{y}\right) / 2$, 计算结果约为 1998.5.

\subsection{2 虚拟传感器尺寸的设置}

虚拟传感器尺寸是捕捉图像的传感器的宽度 和高度, 这决定了物理相机的长宽比. 本文设置虚 拟相机与实际投影机相对应的传感器的分辨率大 小相同, 长为 1024 , 宽为 768 . 具体的设置页面如 图 3 所示.

\begin{tabular}{l|l|}
\hline$\nabla$ Go $\checkmark$ Camera \\
Clear Flags \\
Background \\
Culling Mask
\end{tabular}

图 3 Unity 中虚拟相机的参数设置

\subsection{3 镜头位移的设置}

镜头位移是在对应传感器镜头的水平和垂直 方向上进行调整的. 本文通过改变投影矩阵实现 镜头在水平方向和垂直方向的偏移，即

$$
\begin{gathered}
x=\frac{r_{x} / 2-c_{x}}{r_{x} / 2} \\
y=\frac{c_{y}-r_{y} / 2}{r_{y} / 2}
\end{gathered}
$$

其中, $r_{x}$ 和 $r_{y}$ 分别为所设置虚拟相机分辨率的长和 宽, 即 1024 和 768. 根据式(5)(6)计算出水平及垂直 方向上相对偏移量, 并将上述参数设置为虚拟相机 投影矩阵的 1 行 3 列及 2 行 3 列元素, 作为镜头的 偏移量. 值得注意的是, Unity 中采用的是左手坐 标系，本文系统标定及计算时均采用右手坐标系， 与 Unity 中的虚拟相机的 $y$ 轴是反的, 因此这里 $y$ 值的计算公式与 $x$ 相比相差一个负号. 


\section{3 在虚拟相机中放置孪生数字模型}

根据真实场景中的实物对象，在 3ds Max 中建 立如图 4 所示与真实对象一致的虚拟模型，将其导 人 Unity 虚拟场景中.

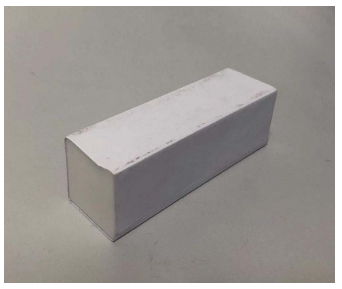

a. 三维打印的实物交互对象

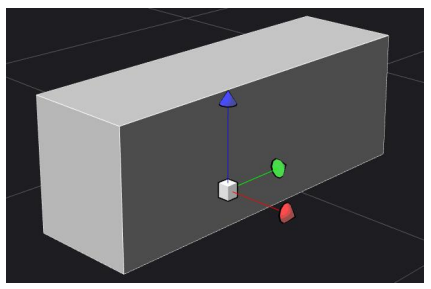

b. 3ds Max 虚拟模型

图 4 孪生建模

用户在使用实物进行交互的过程中，系统实 时检测识别其位姿，根据式(4)计算深度相机坐标 系中识别到的实物对象在投影机坐标系下的位姿， 并在 Unity 中实时调整. 因此, 真实场景中的物体 在用户直接操纵时, 虚拟场景中的模型也会跟随 着用户的移动旋转而呈现同等的变化.

\section{4 投影机显示投影结果}

基于在虚拟场景中建立的与真实场景中实物 对象一致的孪生模型, 调整虚拟相机参数与投影 机一致后, 实现其虚实精确配准. 且通过实时捕获 物理空间中可交互实物的位姿，实时修改虚拟模 型的方向位置与真实场景中一致, 并在 Unity 虚拟 相机渲染需叠加呈现的内容, 最后通过投影机增 强投影在真实场景上，实验的精确配准结果如图 5 所示.

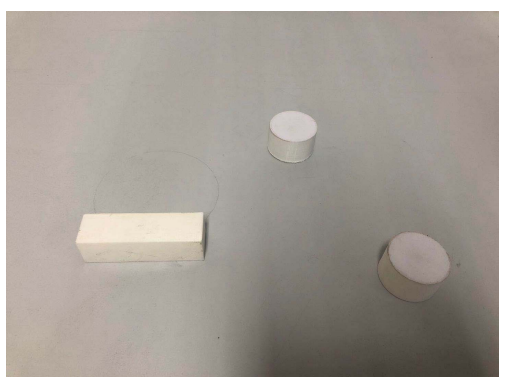

a. 叠加虚拟内容前的三维打印实物交互对象

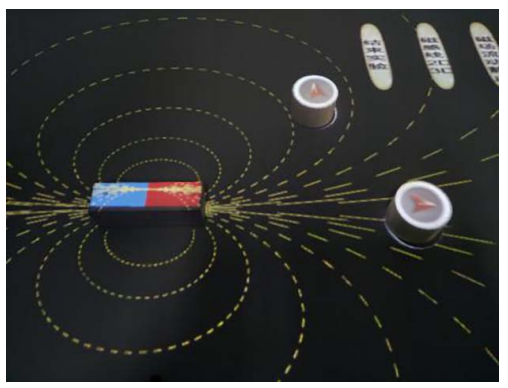

b. 叠加虚拟内容后的三维打印实验交互对象

图 5 精确配准投影显示

\section{3 实验结果与评估}

本文设计并搭建了一套投影式交互桌面系统， 验证所提出的数字孪生法配准技术. 图 6a 所示为 该配准技术设计的实验平台, 图 6b 所示为搭建的 真实实验环境下用户操作的实际场景. 该投影式 交互桌面包括一个普通桌面，具有无高光反射(不 利于深度图获取)、浅色(便于投影画面显示)的特 点; 一个投影机, 型号为 Sharp XG-FN9A, 用于投 影内容, 并输出显示至桌面; 一个微软 Kinect v2.0 传感器, 用于检测并跟踪实物交互对象的位置与 方向; 配置有 Intel Core i7-6700 CPU@ 3.40 GHz 处 理器、16 GB 内存, 装有微软 Windows 8.1 操作系 统、Unity2018.4.5f1 的计算机.

本文以中学物理实验 “认识磁感线” 为例, 设 计了增强现实实验系统, 用创想 CR-5080 三维打 印机及白色打印耗材打印长 $10 \mathrm{~cm}$ 、宽 $3 \mathrm{~cm}$ 、高 $3 \mathrm{~cm}$ 的规则长方体代表磁铁, 直径 $5 \mathrm{~cm}$ 、高 $3 \mathrm{~cm}$ 的圆 柱体代表指南针, 放置于桌面, 系统检测出物体的 位姿，渲染后同步投影磁铁、指南针的纹理以及磁 铁周围分布的磁感线，在磁铁和指南针移动的过 程中, 磁感线的分布和指南针的方向会随之改变.

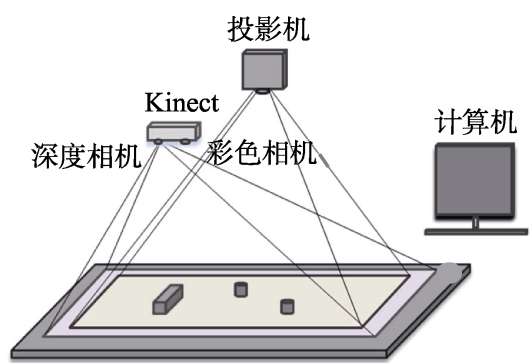

a. 投影式交互桌面设计图

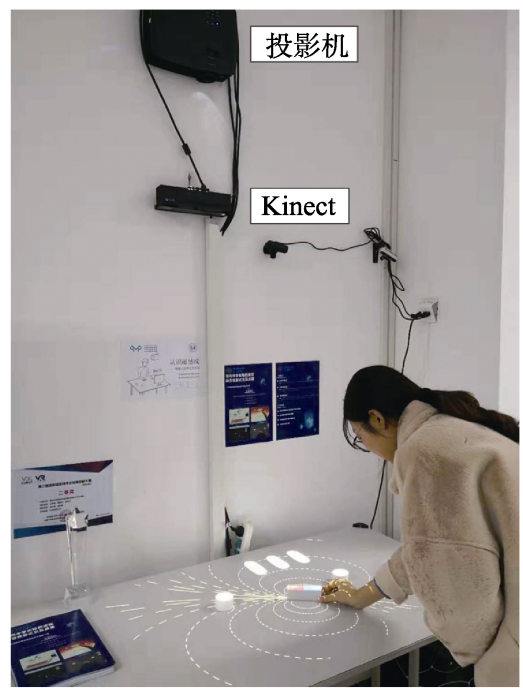

b. 认识磁感线实验实物交互操作

图 6 实验设计与操作图 


\section{1 准确性}

正如文献[12]中提到的，该类系统的精度难以 评估, 主要是因为难以定义什么是精确配准, 即真 实状况值，如用硬件设备进行测量会引人测量设备 的误差. 为了对实验结果中的配准精度进行评估, 本文首先在真实场景中摆放了一个 Intel RealSense D435 相机，利用其 RGB 相机拍摄虚实配准后的场 景, 如图 7b 和图 7d 所示. 又在虚拟场景中添加了 一个与该 RGB 相机内外部参数相同的虚拟相机, 渲染虚拟图像如图 7a 和图 7c 所示. 理论上来说, 这 2 幅图像中磁铁、指南针及磁感线的位置应当完全 一致. 本文在 2 幅对应图像中利用 OpenCV 的 SIFT 特征检测并选取 7 组匹配点, 求解获得对应匹配点 的平均配准检测误差. 所获取图像的分辨率为 $1920 \times 1080$ 像素时, 可以得到平均配准像素误差为 3.87059 , 即对应点的平均欧几里得度量. 第 1 组匹 配图像的水平与垂直方向相对横纵向分辨率的平均 像素误差率分别为 $0.165 \%, 0.228 \%$, 如表 1 所示.
第 2 组匹配图像的水平与垂直方向相对横纵向分辨 率的平均像素误差率分别为 $0.132 \%, 0.274 \%$, 如表 2 所示. 此外, 本文搭建的系统从视觉感受上来说, 没有明显的配准错位，视觉效果好.

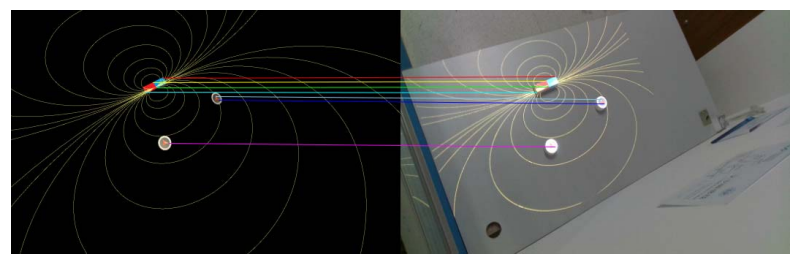

a. 第 1 组虚拟相机渲染图像 b. 第 1 组彩色相机拍摄图像

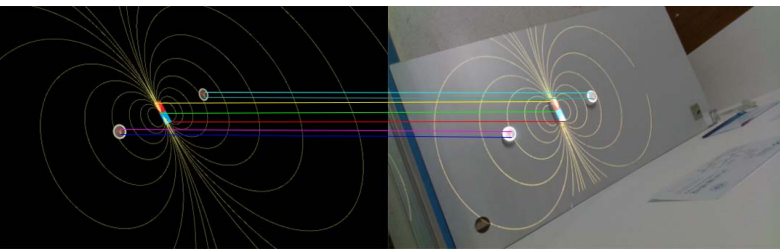

c. 第 2 组虚拟相机渲染图像 d. 第 2 组彩色相机拍摄图像

图 7 虚拟与真实场景中的虚实对比

表 1 第 1 组平均配准像素误差

\begin{tabular}{|c|c|c|c|c|c|}
\hline \multirow{2}{*}{ 匹配点序号 } & \multicolumn{2}{|c|}{ 匹配点坐标 } & \multicolumn{2}{|c|}{ 像素误差率/\% } & \multirow{2}{*}{ 配准检测像素误差 } \\
\hline & 图 7a & 图 7b & 水平方向 & 垂直方向 & \\
\hline 1 & $(207.50,92.75)$ & $(210.75,91.00)$ & 0.169 & 0.171 & 3.69121 \\
\hline 2 & $(194.00,99.25)$ & $(196.75,96.75)$ & 0.143 & 0.231 & 3.71651 \\
\hline 3 & $(199.00,107.25)$ & $(202.75,105.25)$ & 0.195 & 0.185 & 4.25000 \\
\hline 4 & $(183.50,110.75)$ & $(186.75,112.25)$ & 0.169 & 0.138 & 3.57945 \\
\hline 5 & $(280.50,117.25)$ & $(277.75,120.25)$ & 0.143 & 0.277 & 4.06971 \\
\hline 6 & $(284.00,123.25)$ & $(280.25,126.25)$ & 0.195 & 0.277 & 4.80234 \\
\hline 7 & $(215.00,182.75)$ & $(211.75,186.25)$ & 0.169 & 0.324 & 4.77624 \\
\hline 平均值 & & & 0.165 & 0.228 & \\
\hline
\end{tabular}

表 2 第 2 组平均配准像素误差

\begin{tabular}{|c|c|c|c|c|c|}
\hline \multirow{2}{*}{ 匹配点序号 } & \multicolumn{2}{|c|}{ 匹配点坐标 } & \multicolumn{2}{|c|}{ 像素误差率/\% } & \multirow{2}{*}{ 配准检测像素误差 } \\
\hline & 图 7c & 图 7d & 水平方向 & 垂直方向 & \\
\hline 1 & $(283.00,118.25)$ & $(280.75,121.25)$ & 0.117 & 0.278 & 3.75000 \\
\hline 2 & $(282.00,125.25)$ & $(280.25,129.25)$ & 0.091 & 0.370 & 4.36606 \\
\hline 3 & $(213.00,133.25)$ & $(216.25,130.25)$ & 0.169 & 0.278 & 4.42295 \\
\hline 4 & $(220.50,147.25)$ & $(224.75,145.75)$ & 0.221 & 0.139 & 4.50693 \\
\hline 5 & $(237.50,159.50)$ & $(241.75,158.25)$ & 0.221 & 0.116 & 4.43001 \\
\hline 6 & $(167.50,168.75)$ & $(166.75,172.25)$ & 0.039 & 0.324 & 3.57945 \\
\hline 7 & $(167.00,176.25)$ & $(168.25,180.75)$ & 0.065 & 0.417 & 4.67038 \\
\hline 平均值 & & & 0.132 & 0.274 & \\
\hline
\end{tabular}

\section{2 实时性}

在本文硬件平台下，程序运行过程中的平均 帧率为 29.8 帧/s. 通过监视代码, 获取到 50 帧内的 检测与配准所用的时间, 如图 8 所示. 若真实场景
中没有交互动作介人, 则检测配准所用时间稳定 在 $20 \mathrm{~ms}$ 左右, 统计得到平均每帧在实物对象检测 与投影配准中花费的时间为 $22.56 \mathrm{~ms}$, 交互过程无 明显延迟。 


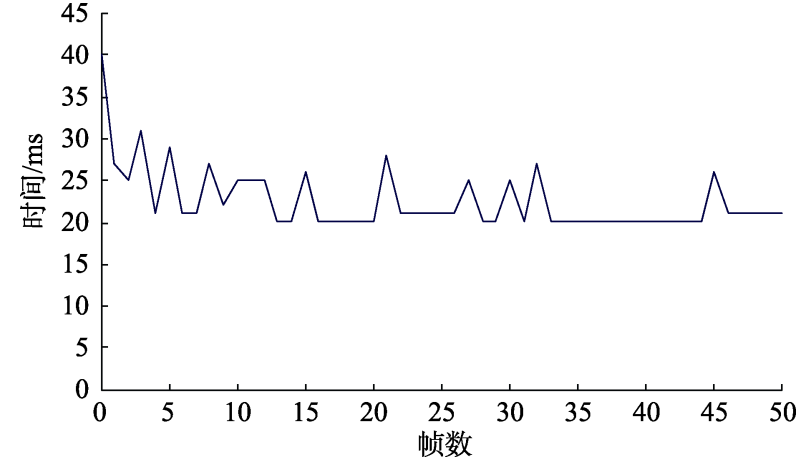

图 8 检测与配准时间

\section{4 结 语}

本文提出一种面向实物交互的空间增强现实 数字孪生法配准技术，通过可触的方式实现物理 世界和数字世界无缝集成，完美契合了增强现实 系统的交互需求 ${ }^{[3]}$. 通过多相机标定与坐标系转 换、深度相机实时捕获交互情境、图像识别、三维 位姿实时检测与恢复等步骤, 实现虚拟内容的精 确投影配准. 实验结果表明, 本文方法配准精度 高, 无明显的视觉误差; 实时性好, 无明显的交互 延迟. 后续工作主要将在以下 2 个方面展开:

（1）目前实验过程没有考虑镜头的畸变，后续 研究中为提高图像处理分析的精度, 畸变必须得 到有效的校正.

(2) 受制于当前使用的 Kinect 深度相机的获 取帧率，系统的更新帧率也在 30 帧/s 左右，后期 考虑更换更高速率的深度相机, 以提高系统的运 行效率.

\section{参考文献(References):}

[1] Bimber O, Raskar R. Spatial augmented reality: merging real and virtual worlds[M]. Boca Raton: AK Peters/CRC Press, 2005: 71-90

[2] Gard N, Hilsmann A, Eisert P. Projection distortion-based object tracking in shader lamp scenarios[J]. IEEE Transactions on Visualization and Computer Graphics, 2019, 25(11): 3105-3113

[3] Bao Hujun, Zhang Guofeng, Qin Xueying. Augmented reality: principles, algorithms and applications[M]. Beijing: Science Press, 2019: 220-288(in Chinese)

(鲍虎军, 章国锋, 秦学英. 增强现实: 原理、算法与应用 [M]. 北京: 科学出版社, 2019: 220-288)

[4] Ishii H, Ullmer B. Tangible bits: towards seamless interfaces between people, bits and atoms[C] //Proceedings of the ACM SIGCHI Conference on Human Factors in Computing System.
New York: ACM Press, 1997: 234-241

[5] Bandyopadhyay D, Raskar R, Fuchs H. Dynamic shader lamps: painting on movable objects[C]//Proceedings of the IEEE and ACM International Symposium on Augmented Reality. Los Alamitos: IEEE Computer Society Press, 2001: 207-216

[6] Marner M R, Thomas B H, Sandor C. Physical-virtual tools for spatial augmented reality user interfaces[C] //Proceedings of the IEEE International Symposium on Mixed and Augmented Reality. Los Alamitos: IEEE Computer Society Press, 2009: 205-206

[7] Khan D, Ullah S, Yan D M, et al. Robust tracking through the design of high quality fiducial markers: an optimization tool for ARToolKit[J]. IEEE Access, 2018, 6: 22421-22433

[8] Fiala M. ARTag, a fiducial marker system using digital techniques[C] //Proceedings of the IEEE Computer Society Conference on Computer Vision and Pattern Recognition. Los Alamitos: IEEE Computer Society Press, 2005: 590-596

[9] Attard G, de Raffaele C, Smith S. TangiBoard: a toolkit to reduce the implementation burden of tangible user interfaces in education[C] //Proceedings of the 13th IEEE International Conference on Application of Information and Communication Technologies. Los Alamitos: IEEE Computer Society Press, 2019: 1-7

[10] Kern J, Weinmann M, Wursthorn S. Projector-based augmented reality for quality inspection of scanned objects[J]. ISPRS Annals of the Photogrammetry, Remote Sensing and Spatial Information Sciences, 2017, IV-2/W4: 83-90

[11] Narita G, Watanabe Y, Ishikawa M. Dynamic projection mapping onto deforming non-rigid surface using deformable dot cluster marker[J]. IEEE Transactions on Visualization and Computer Graphics, 2017, 23(3): 1235-1248

[12] Kagami S, Hashimoto K. Animated stickies: fast video projection mapping onto a markerless plane through a direct closed-loop alignment[J]. IEEE Transactions on Visualization and Computer Graphics, 2019, 25(11): 3094-3104

[13] Kundu S N, Muhammad N, Sattar F. Using the augmented reality sandbox for advanced learning in geoscience education[C] // Proceedings of the IEEE International Conference on Teaching, Assessment, and Learning for Engineering. Los Alamitos: IEEE Computer Society Press, 2017: 13-17

[14] Kurth P, Lange V, Siegl C, et al. Auto-calibration for dynamic multi-projection mapping on arbitrary surfaces[J]. IEEE Transactions on Visualization and Computer Graphics, 2018, 24(11): 2886-2894

[15] Pratticò F G, Baldo $\mathrm{P}$, Cannavò $\mathrm{A}$, et al. Investigating tangible user interaction in mixed-reality robotic games[C] //Proceedings of the IEEE International Conference on Consumer Electronics. Los Alamitos: IEEE Computer Society Press, 2019: 205-210

[16] Zhou Y, Xiao S J, Tang N, et al. Pmomo: projection mapping on movable 3D object[C] //Proceedings of the CHI Conference on Human Factors in Computing Systems. New York: ACM Press, 2016: 781-790

[17] Grieves M W. Product lifecycle management: the new paradigm for enterprises[J]. International Journal of Product Development, 2005, 2(1/2): 71-84 